\title{
Revisiting the links between glycaemia, diabetes and cardiovascular disease
}

\author{
N. Sattar
}

Received: 23 August 2012 /Accepted: 5 December 2012 /Published online: 27 January 2013

(C) Springer-Verlag Berlin Heidelberg 2013

\begin{abstract}
Whilst the interplay between type 2 diabetes and cardiovascular disease (CVD) has been recognised for many years, recent analyses of existing studies have helped refine several aspects of this relationship with relevance to clinical practice. First, recent data demonstrate that fasting glucose is not linearly related to CVD risk in those without diabetes; rather, risk levels escalate (modestly at first) only beyond specific glucose thresholds. Consequently, glucose-based measures may not necessarily enhance CVD risk prediction in those without diabetes. Second, other data confirm that new-onset diabetes is not a post-myocardial infarction 'risk equivalent' state and that, on average, several years of diabetes duration is needed to attain this level of risk. Third, meta-analyses and systemic reviews have confirmed that diabetes increases CVD risk by around twofold on average and this risk is subject to wide variation, being lowest in those newly diagnosed and highest in those with existing vascular disease, proteinuria or renal disease. Fourth, metaanalyses of major glucose-lowering trials suggest that, whilst glucose-lowering lessens vascular risk, risk reduction arising from statins and blood pressure-lowering is greater. Fifth, statins increase diabetes risk, albeit modestly, adding to the emerging concept that some agents that primarily target CVD risk may be diabetogenic, and vice versa. Finally, arising in part from the latter observation, as well as an understanding that CVD and diabetes risk overlap in some individuals but not others, the case for combined CVD/
\end{abstract}

This review was invited based on the Minkowski lecture at the EASD, September 2011.

N. Sattar $(\bowtie)$

Institute of Cardiovascular and Medical Sciences, BHF Glasgow

Cardiovascular Research Centre, University of Glasgow,

126 University Place,

Glasgow G12 8TA, UK

e-mail: naveed.sattar@glasgow.ac.uk diabetes risk screening (generally using non-fasting lipids and $\mathrm{HbA}_{1 \mathrm{c}}$ ), has gained strength.

Keywords Cardiovascular risk $\cdot$ Glucose $\cdot \mathrm{HbA}_{1 \mathrm{c}}$. Linearity $\cdot$ Meta-analysis $\cdot$ Review $\cdot$ Statins $\cdot$ Triacylglycerol

\begin{tabular}{|c|c|}
\hline \\
\hline \multirow[t]{3}{*}{ ADVANCE } & Action in Diabetes and Vascular Disease: \\
\hline & Preterax and Diamicron MR Controlled \\
\hline & Evaluation \\
\hline CVD & Cardiovascular disease \\
\hline eGFR & Estimated GFR \\
\hline ERFC & Emerging Risk Factors Collaboration \\
\hline FDA & Food and Drug Administration \\
\hline FPG & Fasting plasma glucose \\
\hline MI & Myocardial infarction \\
\hline UKPDS & UK Prospective Diabetes Study \\
\hline WOSCOPS & West of Scotland Coronary Prevention Stud \\
\hline
\end{tabular}

\section{Introduction}

This review, based in large part on the Minkoswki Lecture presented in Lisbon 2011, focuses on recent notable gains made in our understanding of the relationship between glycaemia, diabetes and cardiovascular disease (CVD). Rather than being a detailed exposition of the area, it addresses broad concepts using a mixture of meta-analyses of both cohort studies and clinical trials, novel analyses of existing studies, and lateral thinking and data presentations to extend older ideas. In so doing, the work of many knowledgeable colleagues is presented (in addition to my contributions), and it becomes clear that significant gains in knowledge can flow from the sharing of datasets of many studies to yield greater power and, thus, greater precision. The value of strong epidemiological teams working in tandem with 
Fig. 1 HRs for CHD by clinically defined categories of baseline FPG concentration. Analyses were based on 279,290 participants $(14,814$ cases). HRs were adjusted for age, smoking status, BMI, and systolic blood pressure, and, where appropriate, stratified by sex and trial arm. HR $(95 \% \mathrm{CI})$ in people with fasting glucose $5.60-6.99 \mathrm{mmol} / \mathrm{l}$ was 1.12 $(1.06,1.18) . *$ Reference group. Reprinted from [3], with permission from Elsevier

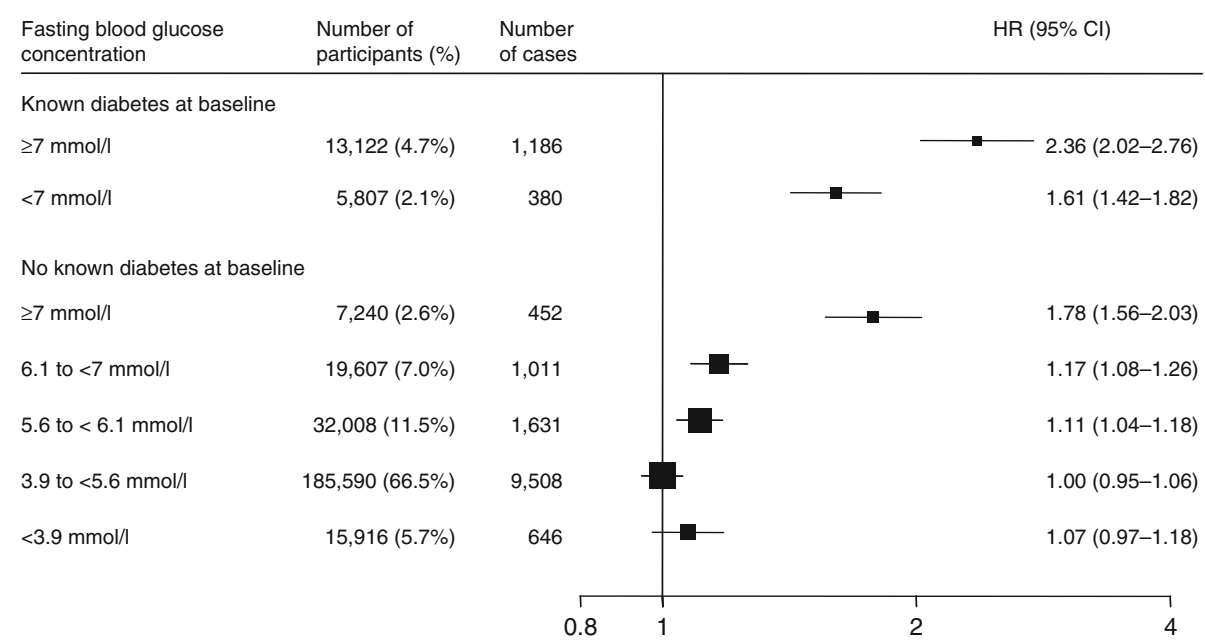

of developing diabetes in men with fasting glucose levels $>5.0 \mathrm{mmol} / \mathrm{l}$ is nearly ninefold that of men with levels $<4.3 \mathrm{mmol} / \mathrm{l}$, even after adjusting for simple risk factors inclusive of age, BMI and blood pressure [1]. However, the relationship between fasting glucose levels and CVD risk in the non-diabetic range has been the subject of uncertainty, with variable reports of linear relationships, threshold effects, no associations or J-shaped associations (reviewed in [2]). This is an important issue, since it would help to determine the value, if any, of using glucose measurements to improve vascular risk prediction. We examined the relationship in a 15 year follow-up of the West of Scotland Coronary Prevention Study (WOSCOPS), during which we observed 2381 CVD events and 1244 deaths, making this, at

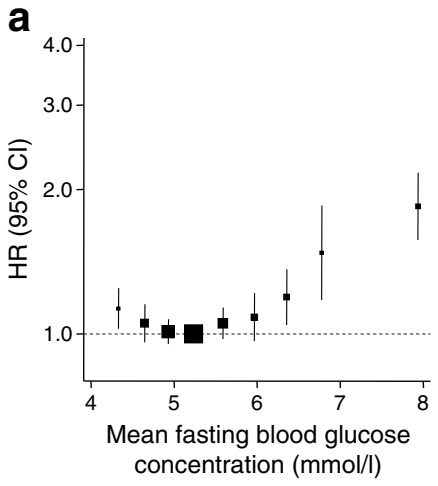

Fig. 2 Comparison of HRs for CHD by long-term average concentrations of FPG concentration (a), total (black squares) and non-HDL(purple squares) cholesterol (b) and systolic blood pressure (c), in a common set of participants. Analyses were done in participants with no known history of diabetes at baseline. Analyses of FPG concentration, total cholesterol and systolic blood pressure were based on 140,624 participants $(10,667$ cases). Analyses of non-HDL-cholesterol were based on a subset of 71,224 participants (4,290 cases). For FPG, participants were classified into groups of baseline fasting concentrations (CHD: $<4.0,4.0, .5,4.5, .0,5.0, .5,5.5, .0,6.0, .5,6.5, .0,7.0, .5$ and $>7.5 \mathrm{mmol} / 1$; ischaemic stroke: $<4.5,4.5, .0,5.0, .5,5.5, .0,6.0, .0$, and $>7.0 \mathrm{mmol} / \mathrm{l}$ ). For the other factors presented, participants were classified according to baseline values as follows: total cholesterol,

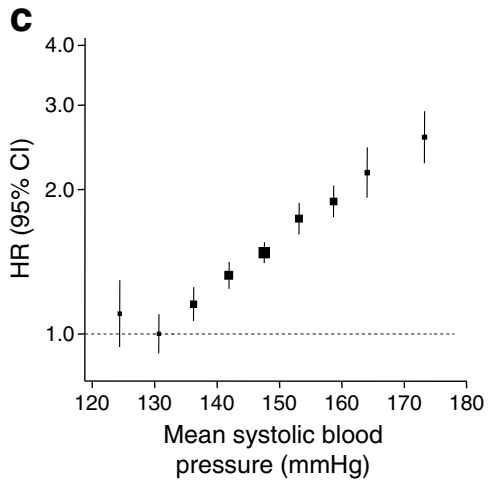

$<4.5,4.5-5.1,5.1-5.7,5.7-6.3,6.3-6.9,6.9-7.5,7.5-8.1,8.1-8.7$, $\geq 8.7 \mathrm{mmol} / \mathrm{l}$; non-HDL-cholesterol, <3.3-3.6, 3.6-4.2, 4.2-4.8, 4.8$5.4,5.4-6.0,6.0-6.6,6.6-7.2, \geq 7.2 \mathrm{mmol} / 1$; systolic blood pressure: $<110,110-120,120-130,130-140,140-150,150-160,160-170$, $170-180, \geq 180 \mathrm{mmHg}$ ). These categories approximately correspond to those used for FPG concentration (i.e. increments of half the SD of each factor). HRs were adjusted, where appropriate, for age, smoking status, BMI, systolic blood pressure, total cholesterol and FPG, and stratified, where appropriate, by sex and trial arm. HRs were plotted against the mean value in each group. Long-term average values were calculated with information from serial measurements. The reference group for each factor is the category with the lowest HR. Reprinted from [3], with permission from Elsevier 
that time, one of the most powerful studies to address this issue [2]. The results showed no significant associations between higher fasting plasma glucose (FPG) within the normal range and incident CVD events or total mortality (most point estimates close to one). That noted, the upper confidence intervals were rather modest, such that more powerful studies were needed to bring us closer to a definitive answer.

The relationship was further investigated in an analysis by the Emerging Risk Factors Collaboration (ERFC) group of investigators, a collaboration that pools individual participant data from multiple cohorts in a robust statistical manner [3]. This study included nearly 700,000 participants with over 50,000 incident vascular outcomes. The results showed that FPG is only modestly and non-linearly (HR $1.17,95 \%$ CI 1.08, 1.26; for FPG 6.1-6.99 vs 3.9-5.59 mmol/1) associated with risk of CVD in the non-diabetic range (Fig. 1). This compared with about a twofold higher CVD risk in diabetic patients. Consequently, FPG did not improve risk prediction metrics for vascular outcomes in those with no history of diabetes. We were also able to show that, whereas FPG concentrations are non-linearly and moderately associated with risk of CHD, total (or non-HDL-) cholesterol levels and systolic blood pressure are more strongly associated with such risk and show a near log-linear relationship (Fig. 2) [3].

$\mathrm{HbA}_{1 \mathrm{c}}$ in the non-diabetic range and CVD risk Having established that FPG levels do not aid vascular risk prediction in those without diabetes, it is worth considering the value of $\mathrm{HbA}_{1 \mathrm{c}}$ for this purpose, especially as a recent meta-analysis demonstrated a somewhat stronger association between $\mathrm{HbA}_{1 \mathrm{c}}$ levels and CHD risk [4]. $\mathrm{HbA}_{1 \mathrm{c}}$ reflects ambient glycaemia for around 3 months and, interestingly, at lower levels is determined to a greater extent by postprandial glycaemia than FPG levels. For $\mathrm{HbA}_{1 \mathrm{c}}$ there are far fewer published studies that report reclassification metrics rather than simple HRs or ORs.

Analysis of the European Prospective Investigation of Cancer (EPIC)-Norfolk study suggested that $\mathrm{HbA}_{1 \mathrm{c}}$ may marginally improve CHD risk reclassification beyond Framingham risk score in men $(3.4 \%, p=0.06)$ but not in women [5]. Subsequent analyses of frozen samples from the Atherosclerosis Risk in Communities (ARIC) cohort extended these findings to suggest that $\mathrm{HbA}_{1 \mathrm{c}}$ improves reclassification of CHD risk (1,198 incident events) in men and women combined (no sex interaction) beyond established predictors by around $2.7 \%$ [6] although, of note, blood pressure was adjusted only as a categorical 'yes/no' variable, which is a potential limitation. A more recent analysis using a German cohort with $>700$ incident events could not confirm a clear reclassification benefit of adding $\mathrm{HbA}_{1 \mathrm{c}}$ to the Systematic Coronary Risk Evaluation Chart (SCORE) for individuals without diabetes mellitus [7]. Hence, this is an area in need of more detailed study as the data are inconclusive at present. It should be noted that as $\mathrm{HbA}_{1 \mathrm{c}}$ escalates in the non-diabetic range, other risk factors also tend to increase. In addition, recently updated CVD risk charts in some countries, such as the UK-based QRISK2 model, now include variables that relate to both glycaemia levels and CVD risk [8]. Hence, by adding ethnicity, social class and BMI, the QRISK2 risk engine may weaken the potential for $\mathrm{HbA}_{1 \mathrm{c}}$ to meaningfully aid CVD risk prediction in those without diabetes [8], and this area clearly needs further work. At present, $\mathrm{HbA}_{1 \mathrm{c}}$ measurement should be used to provide information on diabetes risk or prevalent diabetes status. To this end, a two-stage risk scoring system for diabetes could work well in clinical practice, with a simple risk-assessment score based on simple demographics plus BMI determining which patients should have their $\mathrm{HbA}_{1 \mathrm{c}}$ (or FPG) taken subsequently [9]. Recent National Institute for Health and Clinical Excellence (NICE) guidance on identifying diabetes has made a similar suggestion [10].

\section{Prior hypotheses}

- Glucose/glycaemia measures may aid CVD risk prediction in those without diabetes, beyond established predictors

- Like cholesterol and blood pressure, FPG exhibits approximately log-linear associations with vascular disease throughout the range of its values

\section{New understanding}

- Elevated FPG within the normal range does not aid CVD risk prediction beyond established CVD risk engines

- FPG is not linearly associated with risk of vascular disease in people without diabetes

- Evidence that $\mathrm{HbA}_{1 \mathrm{c}}$ levels (in those without diabetes) meaningfully aid CVD risk prediction is currently inconclusive. Furthermore, recently extended CVD risk engines in certain countries, which contain ethnicity, BMI and social class, lessen the possibility that $\mathrm{HbA}_{1 \mathrm{c}}$ will improve CVD risk prediction in such extended risk scores

\section{Clinical implications}

- Glycaemia-related variables should be measured to ascertain prevalent diabetes or future diabetes risk, whereas CVD risk engines are needed to determine CVD risk in those without diabetes

- $\mathrm{HbA}_{1 \mathrm{c}}$ measurement could currently be restricted to those considered at elevated risk of diabetes on the basis of simple risk tools

- Future studies should address whether $\mathrm{HbA}_{1 \mathrm{c}}$ also aids CVD risk prediction in those without diabetes 


\section{Diabetes as a CHD 'risk equivalent'?}

The seminal paper leading to diabetes being considered as a myocardial infarction (MI) 'risk equivalent' state was published in 1998 [11], an observation pertinent to the timing of statin prescription among diabetic patients. However, considerable confusion and debate continues over the extent to which diabetes mellitus does or does not reflect a CHD risk that is equivalent to that of a previous MI. We tried to reconcile findings in a recent analysis of the British Regional Heart Study [12]. We showed that men who develop diabetes after the age of 60 and have a short duration of diabetes (average of 1.9 years) have a CHD risk around half that of men of similar age who develop diabetes before 60 (average duration $\sim 16.7$ years), with only the latter group having a similar risk to those with previous $\mathrm{MI}$ and no diabetes. In other words, duration of diabetes matters to CHD risk and, typically, a diabetes duration of 8 years or more is needed to reach a $\mathrm{CHD}$ risk equivalent state (Fig. 3). Interestingly, mean duration of diabetes was longer than 8 years in the Haffner et al paper that proposed diabetes as a CHD risk equivalent [11]. By contrast, prospective analysis from Scotland showed CVD mortality risk and risk for hospitalisation for MI to be about threefold lower in persons with newly diagnosed diabetes (mean age 66 years) compared with patients with recent MI [13].

Average excess risk level for vascular disease in diabetes patients? Further data from the ERFC showed that, overall,

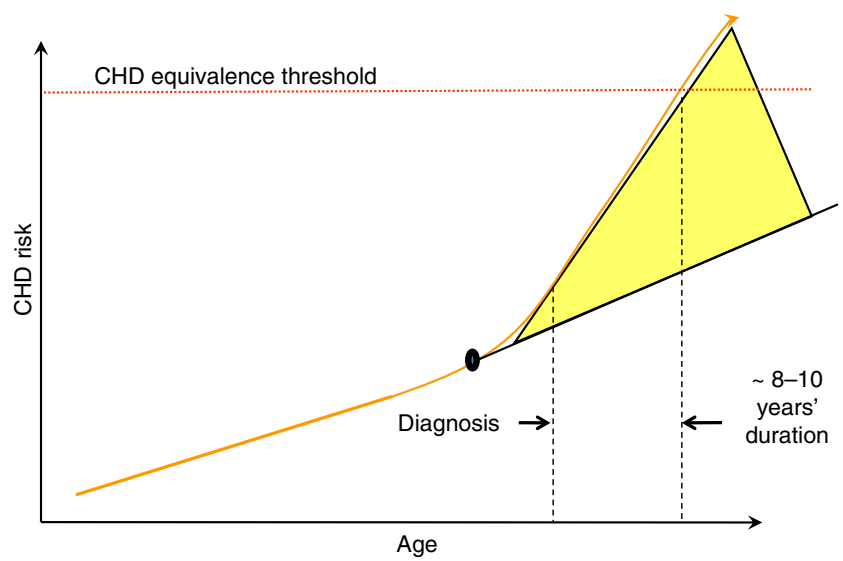

Fig. 3 A conceptual look at vascular risk and its determinants before and during the course of type 2 diabetes. It is important to recognise that CVD risk at the point of diagnosis or conversion to diabetes in the majority of individuals is largely determined by conventional CVD risk factors. After this point, the elevations in glucose into the diabetic range enhance CVD risk gradually over time (coloured area) so that by $8-10$ years' duration patients have attained a CHD risk equivalent state. It is important to recognise that the excess risk indicated by the coloured area may also be contributed to by relative insulin deficiency, as well as hyperglycaemia, or by other, as yet inadequately defined metabolic perturbances linked to type 2 diabetes. In recent years, earlier diabetes diagnosis (combined with lower glycaemia thresholds for diagnosis), and thus earlier use of statins and blood pressure medications have likely further reduced CVD risks in diabetes. Of interest, indirect but powerful evidence for contemporary earlier diagnosis comes from recent Scottish retinopathy data [48]
CVD risk is around twofold greater in diabetic patients compared with people without diabetes, independently of conventional risk factors [3]. There were trends for HRs to be greater in younger people and in women with diabetes [3]. The ERFC analyses were not able to assess the relevance of diabetes duration or renal disease. However, as we showed [12], diabetes duration is important in determining the level of CVD risk, and other recent papers have shown that proteinuria [14] and low estimated GFR (eGFR) $\left(<60 \mathrm{mlmin}^{-1} 1.73 \mathrm{~m}^{-2}\right)$ [15] mark out diabetic patients with excessive CVD risk, with the combination of low eGFR and proteinuria heralding substantially elevated vascular risk [15]. Moreover, it is clear that those with diabetes and CHD are at especially higher CVD risk. Hence, the overall vascular risk of around twofold encapsulates a range of risks; from the relatively low short to medium term vascular risk in patients with newly diagnosed or a short duration of uncomplicated diabetes, to substantially elevated (about four- to fivefold higher HRs) in diabetic patients with existing CHD or proteinuria/low eGFR.

\section{Prior hypothesis}

- Diabetes is a CHD risk equivalent state (i.e. risk levels equivalent to prior MI)

\section{New understanding}

- Diabetes is not a CHD risk equivalent state at diagnosis or in those with short duration of disease (less than about a decade)

- Risk levels approach CHD risk equivalence after a diabetes duration of about a decade or in those with proteinuria or low eGFR

- Diabetic patients with existing CHD have a vascular risk well in excess of those with CHD but without diabetes

\section{Clinical implications}

- Newly diagnosed diabetic patients currently receive statins at lower average CVD risk thresholds than recommended in national guidelines for the general population

- Whilst reintroducing risk scoring in diabetic patients to tailor preventative therapy could be reasonably argued, the current 'fire and forget' approach for statin therapy, whereby statins are recommended for all newly diagnosed type 2 diabetes patients beyond a certain age, has merit and reflects greater lifetime vascular risk trajectories in patients with diabetes

- Most diabetic patients should receive conventional dose statin therapy. Intensive therapy is best targeted to diabetic patients with existing CVD, those with proteinuria or low eGFR. Patients not meeting cholesterol or LDL-cholesterol targets in response to a conventional statin dose may also be considered for intensive statin therapy 


\section{The vascular benefits of lowering glucose in diabetes?}

The mechanism for elevated vascular risk in diabetes remains uncertain but, clearly, elevated glucose must be considered a prime suspect. The seminal UK Prospective Diabetes Study (UKPDS) showed clear benefits of intensive glycaemic control on microvascular risks but failed to show a clear benefit on macrovascular events [16]. Longer term follow-up of UKPDS patients for 10 years after study end, during which differences between $\mathrm{HbA}_{1 \mathrm{c}}$ levels in the trial arms largely disappeared, did show significant reductions in MI for those initially treated with intensive glucose-lowering therapy [17]. These findings have been interpreted as suggesting a 'legacy effect' and, of interest, this concept may fit with the fact that around a decade of diabetes duration is needed to obtain a CHD risk equivalent state.

Of course, several recent studies (Action to Control Cardiovascular Risk in Diabetes [ACCORD], Action In Diabetes And Vascular Disease: Preterax And Diamicron MR Controlled Evaluation [ADVANCE], Veterans' Affairs Diabetes Trial [VADT]) [18-20] have further tested the intensive glycaemic control hypothesis, with some unexpected results necessitating a reappraisal of the hypothesis. We conducted the first meta-analysis of randomised controlled trials that aimed to assess whether more intensive control of glucose reduces long-term CVD clinical events and lengthens lifetime compared with standard treatment [21]. We did so because individual trials may have been underpowered to show clinical benefit. Our analysis demonstrated that a $0.9 \%$ reduction in $\mathrm{HbA}_{1 \mathrm{c}}$ over an average of 5 years of follow-up resulted in a $17 \%$ reduction in non-fatal $\mathrm{MI}$ and a $15 \%$ reduction in CHD events, but with no significant effects on stroke or all-cause mortality. A subsequent high-quality meta-analysis of individual participant data reported similar results and also suggested vascular risk reduction with intensive glycaemic control was more likely in diabetic patients without baseline macrovascular disease [22].

To place the results in context, we also determined that the relative benefits of intensive glycaemia control (i.e. $0.9 \% \mathrm{HbA}_{1 \mathrm{c}}$ reduction) on $\mathrm{CHD}$ risk were clearly more modest than CHD risk reductions seen with, for example, a $1 \mathrm{mmol} / 1$ reduction in LDL-cholesterol or a $4 \mathrm{mmHg}$ reduction in systolic blood pressure (Fig. 4). This observation has been confirmed and built on by Yudkin and Richter [23] and it is now generally accepted that statin therapy and blood pressure control are relatively more important than targeting glycaemia (by proven therapies) to lessen CVD risk. The long-term results of the Steno-2 study also confirm the importance

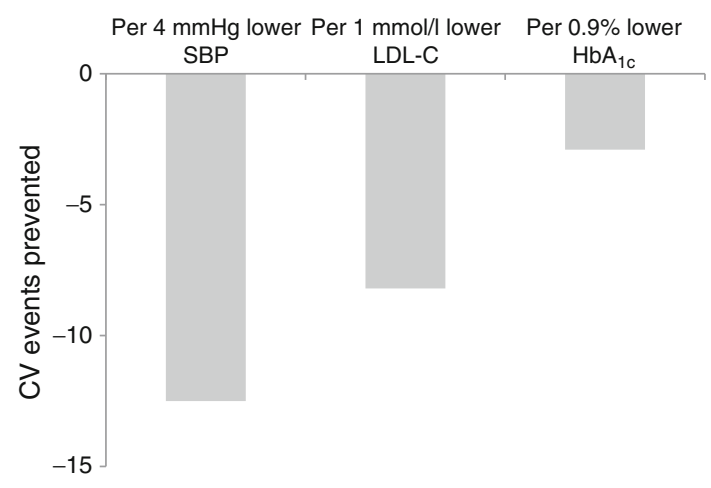

Fig. 4 A paradigm shift for CVD prevention in diabetes? Benefit of different interventions per 200 diabetic patients treated for 5 years. This figure, based on data discussed in [20], albeit the three estimates derived from different patient populations, shows that vascular benefits from lipid-lowering with statins and BP lowering are considerably greater than those achieved from glucose-lowering in patients with type 2 diabetes. The same broad conclusion has been reached by others [22], contributing to a paradigm shift in our understanding of the best current methods for CVD risk reduction in diabetes. LDL-C, LDLcholesterol; SBP, systolic BP

of multi-factorial intervention in diabetes, with particular focus on established risk factors [24]. Of course, whether newer glucose-lowering therapies, with lower risks of hypoglycaemia and better weight-change profiles, have better macrovascular protective properties is the subject of several ongoing CVD endpoint trials.

$H b A_{1 c}$ and CVD risk in diabetes? Is there a threshold effect? Whilst the UKPDS data originally suggested vascular risk increases from relatively modest $\mathrm{HbA}_{1 \mathrm{c}}$ levels (of $\sim 5.5 \%, 37 \mathrm{mmol} / \mathrm{mol}$ ), recent analyses of the ADVANCE study challenged this finding [25]. In ADVANCE, microvascular risk appeared to increase only once $\mathrm{HbA}_{1 \mathrm{c}}$ exceeded $6.5 \%$ (48 $\mathrm{mmol} / \mathrm{mol}$ ), while CVD risk increased when $\mathrm{HbA}_{1 \mathrm{c}}$ exceeded $7.0 \%(53 \mathrm{mmol} / \mathrm{mol})$. Risk associations appeared to be relatively weak below these thresholds [25]. Although these are post hoc analyses of trial data, they may partially explain the recent neutral results of the Outcome Reduction with Initial Glargine Intervention (ORIGIN) study [26]. Here, basal insulin was used to bring down fasting glucose to normal levels in participants with cardiovascular risk factors plus impaired fasting glucose, impaired glucose tolerance or type 2 diabetes, but it was notable that the median $\mathrm{HbA}_{1 \mathrm{c}}$ at baseline in both ORIGIN glycaemia arms was only $6.4 \%(46 \mathrm{mmol} / \mathrm{mol})$, which is well below the threshold of $7.0 \%$ ( $53 \mathrm{mmol} / \mathrm{mol})$ for CVD risk suggested by ADVANCE data. In other words, CVD endpoint trials of glycaemia-lowering agents may be best conducted in diabetic patients with an $\mathrm{HbA}_{1 \mathrm{c}}$ level well above $7.0 \%(53 \mathrm{mmol} / \mathrm{mol})$. Of course, recruited patients need to have a high CVD risk or prevalent disease for the study to have sufficient power. 


\section{Prior hypothesis}

- Targeting glycaemia levels in diabetes is critical to lower CVD risk

- Reducing glycaemia levels in diabetes to near normal ranges will provide added vascular benefit

\section{Newer understanding}

- Whilst targeting glycaemia lowers CVD risk, it does so only modestly. There is stronger evidence for benefits of glycaemic control on retinopathy risks

- Blood pressure treatments and lowering LDL-cholesterol with statins lessens CHD risks to greater extents than glucose-lowering in patients with diabetes

- Reducing glucose levels in diabetic patients with only modestly elevated glycaemia levels at baseline (e.g. $\mathrm{HbA}_{1 \mathrm{c}} 6.5-7.0 \%$ or $48-53 \mathrm{mmol} / \mathrm{mol}$ ) may not necessarily achieve any vascular benefit, at least within the short to medium term

\section{Clinical implications}

- To reduce CVD risk, lipid-lowering with statins and blood pressure control must be prioritised early in diabetes management

\section{Triacylglycerol levels: stronger indicators of metabolic dysregulation/ectopic fat vs vascular risk?}

Elevated LDL-cholesterol is well recognised as a causal risk factor for CHD and, based on robust trial evidence, statins are now the mainstay of lipidlowering for prevention of CVD. However, whilst cholesterol targets can be reached in the majority of our patients, many continue to have elevated triacylglycerol levels, but the degree to which this represents an additional independent CVD risk factor remains in doubt. To resolve this issue, we related lipids to CVD risk in a meta-analysis of prospective studies that was based on individual participant data [27]. This work showed that whilst elevated cholesterol and low HDL-cholesterol predicted higher CVD risk in age, sex and other lipid-variable-adjusted models, triacylglycerol levels were not related to risk for vascular events once non-HDL-cholesterol and HDL-cholesterol were accounted for. The results were similar in patients with or without diabetes. As a result, we recommended that lipid assessment in vascular disease can be simplified by measurement of total and HDL-cholesterol levels without the need to fast and without regard to triacylglycerol [27]. Of course, this epidemiological work does not necessarily mean that triacylglycerol is not causally related to vascular risk, which is a separate question requiring different approaches. Nevertheless, it is of interest to note that recent fibrate trials failed to show a benefit in the prevention of vascular events in diabetic patients. The results have led the US Food and Drug Administration (FDA) to recommend a need for further large-scale trials to allow definitive conclusions in this area, but for now fibrates are not routinely recommended [28].

What is perhaps less well perceived is that for a given elevation in triacylglycerol in people without diabetes, a given increment in triacylglycerol is a stronger risk factor for diabetes as compared with its weaker associations with vascular risk [29]. This is partly because, in the majority, elevated plasma triacylglycerol levels represent another manifestation of ectopic fat whereby excess fat is being deposited in the plasma pool, in the same way as excess fat appears in the liver in many patients at risk for type 2 diabetes, or with prevalent diabetes [30]. Furthermore, and as most physicians recognise, triacylglycerol levels can be markedly elevated when patients have poor diabetes control, with rapid improvement possible with an appropriate reduction in glycaemia levels.

Furthermore, and as noted previously, as with triacylglycerol, FPG in the non-diabetic range is also a far stronger risk factor for type 2 diabetes than for CVD [1]; the same association also holds true for current BMI [29]. As a result, in a novel analysis we showed that the metabolic syndrome criteria are more aligned with diabetes than with CVD risk, and consequently have little clinical value [31], an observation now widely accepted [32].

Of course, fibrates are still often used in patients with diabetes who have modest elevations in triacylglycerol (typically greater than about $5-10 \mathrm{mmol} / \mathrm{l}$ ), often to mitigate against pancreatitis risk. However, in our recent meta-analysis of lipid-lowering trials, statins, not fibrates, were associated with a lower risk of pancreatitis [33]. This work suggests that statins should be the first-line treatment for patients with modest triacylglycerol elevations for prevention of pancreatitis. It also suggests a need for trials to investigate the effects of fibrates vs statins on pancreatitis risk in those with significant hypertriglyceridaemia. 


\section{Prior hypothesis}

- Triacylglycerol levels are strong risk factors for CVD

\section{Newer understanding}

- Triacylglycerol levels are not associated with CVD risk independently of established predictors

- Triacylglycerol levels are more strongly aligned with diabetes risk and, in the majority of patients, represent another clinical example of 'ectopic fat'

- Statins, not fibrates, lowered pancreatitis risk in clinical trials of lipid-lowering agents

\section{Clinical implications}

- Lipid assessment for vascular risk can be done in the non-fasting state

- The metabolic syndrome criteria (which contain FPG, triacylglycerol and BMI) are more aligned with diabetes risk and, as a result, have no benefit in clinical practice - In patients with modest elevations of triacylglycerol, statins appear better supported by available data than fibrates for preventing pancreatitis

- There is currently little evidence to support targeting minor elevations in triacylglycerol levels in patients established on statins for prevention of CVD

\section{Diabetes and vascular risks in women vs men}

It is well known that women with diabetes have a greater relative excess CVD risk compared with men. This was most recently confirmed in the ERFC analysis, in which the HR for CVD in women was 2.59 (95\% CI 2.29, 2.93) vs 1.89 (1.73, 2.06) in men [3]. However, the mechanisms behind this observation remain to be fully established. We hypothesised and confirmed that whilst women without diabetes have more favourable cardiovascular risk factor levels than men, many of these advantages are attenuated or lost in women with diabetes [34]. In other words, CVD risk factors change much more between non-diabetes and diabetes in women compared with men. Of interest, these greater CVD risk factor changes were generally explained by the greater increase in adiposity and insulin resistance associated with diabetes in women than in men. Our results were therefore consistent with the hypothesis that women have to undergo a greater metabolic deterioration to develop diabetes than men do. In a subsequent paper using data from the Scottish Diabetes Registry, we developed this theme further by demonstrating that women have to attain a greater BMI than men do to be diagnosed with diabetes across nearly the entire adult age profile, with the BMI differential being most marked at younger ages [35]. Such work therefore confirmed and extended prior observations from others [36], and we believe it did so in a novel and comprehensible manner. Of interest, others [37] have recently confirmed our findings from analysis of Scottish data [35].

\section{Prior observations}

- Women with diabetes have a greater relative excess CVD risk compared with men

- Women with diabetes tend to be heavier than their male counterparts

\section{Newer understanding}

- Women have to attain a greater BMI to develop type 2 diabetes than men do, especially at younger ages, and, as a result, CVD risk factors linked to adiposity/insulin resistance worsen more in women as they transition to diabetes

- This observation may also explain the higher prevalence of diabetes in middle-aged men compared with women in many parts of the world, since population BMI levels are generally comparable between men and women

\section{Clinical implications}

-Women with diabetes should receive similar risk factor treatment as men for prevention of CVD

- Men develop diabetes at a lower average BMI, but less often put themselves forward for weight loss interventions. More men should be better targeted for weight loss/ lifestyle interventions for prevention of diabetes (and perhaps at a lower average BMI) and barriers to their uptake of interventions need to be identified

\section{Statins and diabetes risks}

Statins unequivocally lower CVD risk across all risk thresholds and do so in a dose-dependent manner. A post hoc analysis from the WOSCOPS group suggested pravastatin may also lessen diabetes risk, and a number of mechanisms for this suggestion were postulated [38]. However, this finding was not confirmed in the JUPITER study which reported a higher, not lower, risk of diabetes with rosuvastatin compared with placebo [39]. To resolve uncertainties, we conducted two meta-analyses of randomised trials. The first pooled published 
and unpublished data from 13 placebo- and standard carecontrolled trials and demonstrated a $9 \%$ increased risk for diabetes $(95 \%$ CI $2 \%, 17 \%)$ for statin recipients [40], whereas the second analysed data from the five intensive vs conventional dose statin studies and reported a $12 \%$ higher risk $(95 \%$ CI 4\%,22\%) for those on intensive therapy [41]. These two studies provide the most robust evidence that statins increase diabetes risk, a finding recently added to the statin label by the FDA. In absolute terms, we determined that treatment of 255 $(95 \%$ CI 150,852$)$ patients with statins for 4 years resulted in one extra case of diabetes [40]. We were careful in both papers to point out that clinical practice for patients with moderate or high cardiovascular risk or existing CVD should not change $[40,41]$. There are some clinical implications, however. First, patients without diabetes should be informed of the potential of an increased risk of diabetes with statin use and, as a result, told that they must not forego lifestyle improvements. Given the modest potential increase in diabetes risk with statins, patients taking these agents should be encouraged to control their weight and improve their level of activity. Second, we believe the findings strengthen the need to undertake combined CVD and diabetes risk screening. We have previously suggested this can be undertaken in the majority of patients using non-fasting measurement of lipids and $\mathrm{HbA}_{1 \mathrm{c}}$, where the lipid data are collated with other CVD risk factors to determine CVD risk, whereas the $\mathrm{HbA}_{1 \mathrm{c}}$ value defines diabetes risk [42]. $\mathrm{HbA}_{1 \mathrm{c}}$ measurement may also be usefully repeated after a few months post statin commencement.

\section{Prior hypothesis}

- Statins, by virtue of their lipid-lowering and apparent pleiotropic effect, may lessen diabetes risk

\section{New understanding}

- Statins increase the risk of diabetes, albeit modestly, and do so in a dose-dependent manner

\section{Clinical implications}

- These findings indicate that there should not be a change of policy of prescribing statins to diabetes patients, to those in the secondary prevention category, or those at moderate or elevated vascular risk. However, in all patients without diabetes, the statin risk is usefully mentioned as a way to further incentivise the need for positive lifestyle changes

- This finding also increases the impetus to undertake combined CVD/diabetes screening

- Future trials of lipid-lowering agents should include new-onset diabetes as an endpoint, in the same way that many trials of newer diabetes agents are assessing their effects on CVD risk

\section{Future patterns of vascular disease in patients with diabetes?}

There is now clear evidence of declining mortality rates in diabetes (compared with non-diabetes) over several decades, an observation we confirmed in a recent ERFC analysis [43]. Furthermore, US data analyses suggest a continuing reduction in CVD mortality rates in diabetes in recent years [44], explicable in large part to better management of CVD risk factors [45]. Ongoing trials with newer glucoselowering agents (which cause far less hypoglycaemia and weight gain) may add further CVD benefit so that, at least for the foreseeable future, CVD rates may continue to decline. This noted, as CVD death rates go down, more patients with type 2 diabetes are living longer than ever and this may present newer problems, including rising levels of cognitive decline or, potentially, greater heart failure and cancer rates. However, at the same time, there is an increasing population of younger, more obese patients with type 2 diabetes [46], a group likely to have more rapid glycaemia progression, as well as a general rise in type 2 diabetes rates worldwide [47], such that diabetes will increasingly account for a higher percentage of the total CVD burden in society. These shifting patterns of disease will present unique problems in the future.

Acknowledgements Whilst I have been involved in many of the papers discussed herein, the leading role of many excellent colleagues deserves mention. I would like to thank D. Preiss (University of Glasgow) for his critical reading of this paper and his expert work on several joint papers. I also wish to thank colleagues from the Emerging Risk Factor Collaboration, including, in particular, J. Danesh and E. Di Angelantonio (both University of Cambridge), for their excellent leadership of this important collaboration. Thanks also to G. Wannamethee (University College London), for allowing me to collaborate on novel data analyses of the British Regional Heart Study, to K. Ray (St George's Hospital, London), for his expertise in the conduct of collaborative metaanalyses, and to N. Forouhi (MRC Epidemiology Unit, Cambridge) for many helpful discussions on aspects relevant to the current review. D. Lawlor (University of Bristol) is also credited for sharing data and for helping shape relevant papers. Finally, thanks to my many colleagues in the Scottish Diabetes Research Network Epidemiology group for allowing us to test hypotheses in this rich dataset, and to many other Glasgow colleagues for their excellent collaborations on several ideas of mutual interest.

Funding I wish to acknowledge grant support from Diabetes UK, British Hearth Foundation, Chest Heart Stroke, Scotland and the Chief Scientist Office, Scotland in supporting the conduct of relevant work.

Duality of interest I have consulted, or been on the speakers' bureau, for Astrazeneca, Bristol Myers Squibb, Eli-Lilly/Amgen, MSD, Roche and Sanofi-Aventis. I have also received grant support from Merck and Pfizer.

Contribution statement The author was responsible for the conception, design and drafting of the manuscript, and approved the final version for publication. 


\section{References}

1. Freeman DJ, Norrie J, Caslake MJ et al (2002) C-reactive protein is an independent predictor of risk for the development of diabetes in the West of Scotland Coronary Prevention Study. Diabetes 51:1596-1600

2. Preiss D, Welsh P, Murray HM et al (2010) Fasting plasma glucose in non-diabetic participants and the risk for incident cardiovascular events, diabetes, and mortality: results from WOSCOPS 15-year follow-up. Eur Heart J 31:1230-1236

3. Emerging Risk Factors Collaboration, Sarwar N, Gao P et al (2010) Diabetes mellitus, fasting blood glucose concentration, and risk of vascular disease: a collaborative meta-analysis of 102 prospective studies. Lancet 375:2215-2222

4. Sarwar N, Aspelund T, Eiriksdottir G, et al (2010) Markers of dysglycaemia and risk of coronary heart disease in people without diabetes: Reykjavik prospective study and systematic review. PLoS Med 7:e1000278

5. Simmons RK, Sharp S, Boekholdt SM et al (2008) Evaluation of the Framingham risk score in the European Prospective Investigation of Cancer-Norfolk cohort: does adding glycated hemoglobin improve the prediction of coronary heart disease events? Arch Intern Med 168:1209-1216

6. Selvin E, Steffes MW, Zhu H et al (2010) Glycated hemoglobin, diabetes, and cardiovascular risk in nondiabetic adults. $\mathrm{N}$ Engl $\mathrm{J}$ Med 362:800-811

7. Schöttker B, Müller H, Rothenbacher D, Brenner H (2013) Fasting plasma glucose and $\mathrm{HbA}_{1 \mathrm{c}}$ in cardiovascular risk prediction: a sex-specific comparison in individuals without diabetes mellitus. Diabetologia 56:92-100

8. Hippisley-Cox J, Coupland C, Vinogradova Y et al (2008) Predicting cardiovascular risk in England and Wales: prospective derivation and validation of QRISK2. BMJ 336:1475-1482

9. Wannamethee SG, Papacosta O, Whincup PH et al (2011) The potential for a two-stage diabetes risk algorithm combining nonlaboratory-based scores with subsequent routine non-fasting blood tests: results from prospective studies in older men and women. Diabet Med 28:23-30

10. NICE (2012) Preventing type 2 diabetes: risk identification and interventions for individuals at high risk. NICE public health guidance 38. Available from www.nice.org.uk/nicemedia/live/ 13791/59951/59951.pdf. Accessed 3 Jan 2013

11. Haffner SM, Lehto S, Rönnemaa T, Pyörälä K, Laakso M (1998) Mortality from coronary heart disease in subjects with type 2 diabetes and in nondiabetic subjects with and without prior myocardial infarction. N Engl J Med 339:229-234

12. Wannamethee SG, Shaper AG, Whincup PH, Lennon L, Sattar N (2011) Impact of diabetes on cardiovascular disease risk and all-cause mortality in older men: influence of age at onset, diabetes duration, and established and novel risk factors. Arch Intern Med 171:404-410

13. Evans JM, Wang J, Morris AD (2002) Comparison of cardiovascular risk between patients with type 2 diabetes and those who had had a myocardial infarction: cross sectional and cohort studies. BMJ 324:939-942

14. Preiss D, Sattar N, McMurray JJ (2011) A systematic review of event rates in clinical trials in diabetes mellitus: the importance of quantifying baseline cardiovascular disease history and proteinuria and implications for clinical trial design. Am Heart J 161:210-219

15. Tonelli M, Muntner P, Lloyd A et al (2012) Risk of coronary events in people with chronic kidney disease compared with those with diabetes: a population-level cohort study. Lancet 380:807-814

16. UK Prospective Diabetes Study (UKPDS) Group (1998) Intensive blood-glucose control with sulphonylureas or insulin compared with conventional treatment and risk of complications in patients with type 2 diabetes (UKPDS 33). Lancet 352:837-853
17. Holman RR, Paul SK, Bethel MA, Neil HA, Matthews DR (2008) Long-term follow-up after tight control of blood pressure in type 2 diabetes. N Engl J Med 359:1565-1576

18. The Action to Control Cardiovascular Risk in Diabetes Study Group (2008) Effects of intensive glucose lowering in type 2 diabetes. N Engl J Med 358:2545-2559

19. The ADVANCE Collaborative Group (2008) Intensive blood glucose control and vascular outcomes in patients with type 2 diabetes. N Engl J Med 358:2560-2572

20. Duckworth W, Abraira C, Moritz T et al (2009) Glucose control and vascular complications in veterans with type 2 diabetes. $\mathrm{N}$ Engl J Med 360:129-139

21. Ray KK, Seshasai SR, Wijesuriya S et al (2009) Effect of intensive control of glucose on cardiovascular outcomes and death in patients with diabetes mellitus: a meta-analysis of randomised controlled trials. Lancet 373:1765-1772

22. Control Group, Turnbull FM, Abraira C et al (2009) Intensive glucose control and macrovascular outcomes in type 2 diabetes. Diabetologia 52:2288-2298

23. Yudkin JS, Richter B (2009) Intensive glucose control and cardiovascular outcomes. Lancet 374:522

24. Gaede P, Lund-Andersen H, Parving HH, Pedersen O (2008) Effect of a multifactorial intervention on mortality in type 2 diabetes. N Engl J Med 358:580-591

25. Zoungas S, Chalmers J, Ninomiya T et al (2012) Association of $\mathrm{HbA}_{1 \mathrm{c}}$ levels with vascular complications and death in patients with type 2 diabetes: evidence of glycaemic thresholds. Diabetologia 55:636-643

26. ORIGIN Trial Investigators, Gerstein HC, Bosch J et al (2012) Basal insulin and cardiovascular and other outcomes in dysglycemia. N Engl J Med 367:319-328

27. Emerging Risk Factors Collaboration, Di Angelantonio E, Sarwar $\mathrm{N}$ et al (2009) Major lipids, apolipoproteins, and risk of vascular disease. JAMA 302:1993-2000

28. Goldfine AB, Kaul S, Hiatt WR (2011) Fibrates in the treatment of dyslipidemias - time for a reassessment. N Engl J Med 365: $481-484$

29. Sattar N (2008) Why metabolic syndrome criteria have not made prime time: a view from the clinic. Int J Obes (Lond) 32(Suppl 2): $\mathrm{S} 30-\mathrm{S} 34$

30. Ghouri N, Preiss D, Sattar N (2010) Liver enzymes, nonalcoholic fatty liver disease, and incident cardiovascular disease: a narrative review and clinical perspective of prospective data. Hepatology 52:1156-1161

31. Sattar N, McConnachie A, Shaper AG et al (2008) Can metabolic syndrome usefully predict cardiovascular disease and diabetes? Outcome data from two prospective studies. Lancet 371:1927-1935

32. Simmons RK, Alberti KG, Gale EA et al (2010) The metabolic syndrome: useful concept or clinical tool? Report of a WHO Expert Consultation. Diabetologia 53:600-605

33. Preiss D, Tikkanen MJ, Welsh P et al (2012) Lipid-modifying therapies and risk of pancreatitis. A meta-analysis. JAMA 304:804-811

34. Wannamethee SG, Papacosta O, Lawlor DA et al (2012) Do women exhibit greater differences in established and novel risk factors between diabetes and non-diabetes than men? The British Regional Heart Study and British Women's Heart Health Study. Diabetologia 55:80-87

35. Logue J, Walker JJ, Colhoun HM et al (2011) Do men develop type 2 diabetes at lower body mass indices than women? Diabetologia 54:3003-3006

36. Sourij H, Holman RR (2012) Women develop diabetes at higher BMIs than men. Diabetologia 55:855-856

37. Paul S, Thomas G, Majeed A, Khunti K, Klein K (2012) Women develop type 2 diabetes at a higher body mass index than men. Diabetologia 55:1556-1557 
38. Freeman DJ, Norrie J, Sattar N et al (2001) Pravastatin and the development of diabetes mellitus: evidence for a protective treatment effect in the West of Scotland Coronary Prevention Study. Circulation 103:357-362

39. Ridker PM, Danielson E, Fonseca FA et al (2008) Rosuvastatin to prevent vascular events in men and women with elevated Creactive protein. N Engl J Med 359:2195-2207

40. Sattar N, Preiss D, Murray HM et al (2010) Statins and risk of incident diabetes: a collaborative meta-analysis of randomised statin trials. Lancet 375:735-742

41. Preiss D, Seshasai SR, Welsh P et al (2011) Risk of incident diabetes with intensive-dose compared with moderate-dose statin therapy: a meta-analysis. JAMA 305:2556-2564

42. Preiss D, Khunti K, Sattar N (2011) Combined cardiovascular and diabetes risk assessment in primary care. Diabet Med 28:19-22

43. Emerging Risk Factors Collaboration, Seshasai SR, Kaptoge S et al (2011) Diabetes mellitus, fasting glucose, and risk of causespecific death. N Engl J Med 364:829-841
44. Gregg EW, Cheng YJ, Saydah S et al (2012) Trends in death rates among U.S. adults with and without diabetes between 1997 and 2006: findings from the National Health Interview Survey. Diabetes Care 35:1252-1257

45. Ford ES (2011) Trends in the risk for coronary heart disease among adults with diagnosed diabetes in the U.S.: findings from the National Health and Nutrition Examination Survey, 1999-2008. Diabetes Care 34:1337-1343

46. Harron KL, Feltbower RG, McKinney PA, Bodansky HJ, Campbell FM, Parslow RC (2011) Rising rates of all types of diabetes in south Asian and non-south Asian children and young people aged 0-29 years in West Yorkshire, UK, 1991-2006. Diabetes Care 34:652-654

47. Wild S, Roglic G, Green A, Sicree R, King H (2004) Global prevalence of diabetes: estimates for the year 2000 and projections for 2030. Diabetes Care 27:1047-1053

48. Looker HC, Nyangoma SO, Cromie D et al (2012) Diabetic retinopathy at diagnosis of type 2 diabetes in Scotland. Diabetologia 55:2335-2342 